\title{
Morphology Control in the Hydrothermal Synthesis of FeS Nanoplate- lets
}

Melonie P. Thomas, Ahamed Ullah, Rose H. Pham, Honore Djieutedjeu, John P. Selegue, and Beth S. Guiton*

Department of Chemistry, University of Kentucky, Lexington; KY. 40506

*: Corresponding author: beth.guiton@uky.edu 
$\mathrm{HS}^{-}(\mathrm{aq})+\mathrm{OH}^{-}(\mathrm{aq}) \rightarrow \mathrm{S}(\mathrm{s})+\mathrm{H}_{2} \mathrm{O}(\mathrm{l})+2 \mathrm{e}^{-}(\mathrm{pH}>7)$

$\mathrm{Fe}^{3+}(\mathrm{aq})+\mathrm{e}^{-} \rightarrow \mathrm{Fe}^{2+}(\mathrm{aq})$

Balanced equation:

$2 \mathrm{Fe}^{3+}(\mathrm{aq})+\mathrm{HS}^{-}(\mathrm{aq})+\mathrm{OH}^{-}(\mathrm{aq}) \rightarrow 2 \mathrm{Fe}^{2+}(\mathrm{aq})+\mathrm{S}(\mathrm{s})+\mathrm{H}_{2} \mathrm{O}(\mathrm{l})$

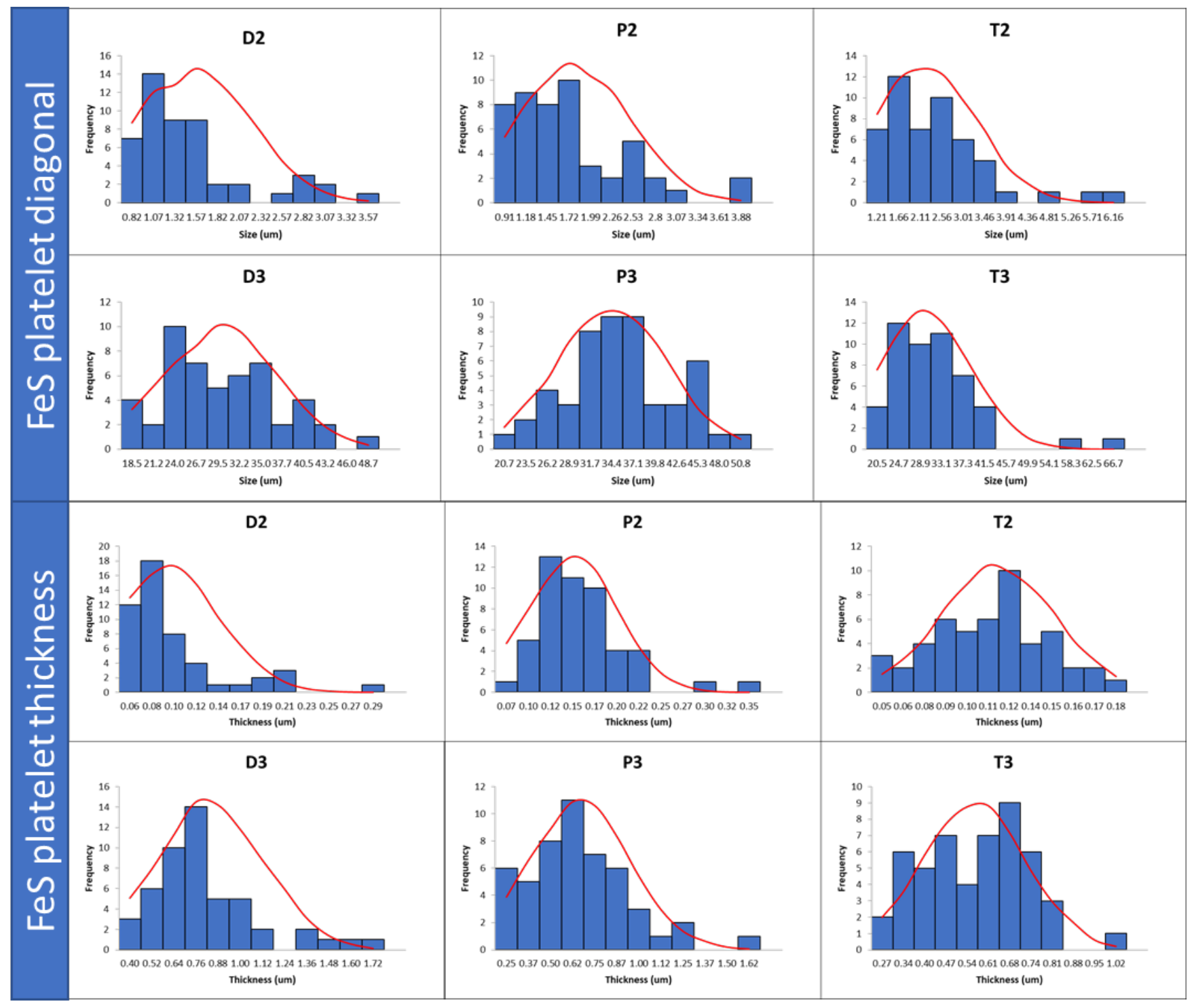

Figure S1. The platelet diagonal and thickness distribution histograms of the FeS platelets calculated by ImageJ $($ software. 

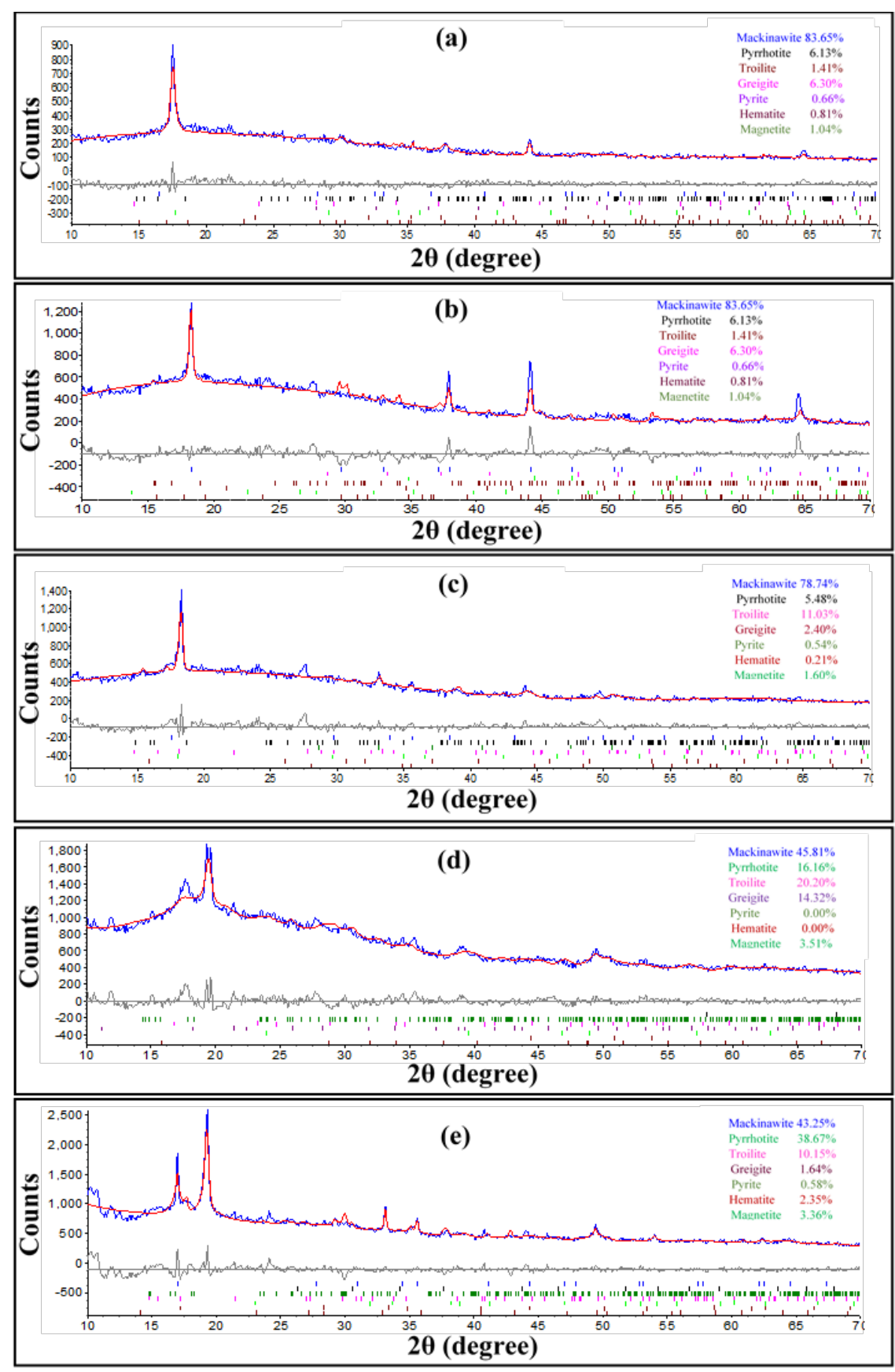

Figure S2. (a) $\mathrm{FeCl}_{3}$ DETA, (b) $\mathrm{FeCl}_{2}$ TEPA, (c) $\mathrm{FeCl}_{3}$ TEPA, (d) $\mathrm{FeCl}_{2}$ TETA and (e) $\mathrm{FeCl}_{3}$ TETA. The solid blue, red and gray lines represent the observed, calculated and difference profiles, respectively. The vertical bars (|) indicate the positions of Bragg reflections of the calculated patterns of different iron sulfide and oxide phases. 


\section{Rietveld Analysis of Powder X-ray Diffraction Data}

Rietveld analysis of iron sulfides samples was performed using Topas software package version 4.2 (Bruker). Refinement of the powder X-ray diffraction pattern of the iron sulfides displays that mackinawite (FeS) phase is the predominant phase in all samples. In addition, refinement also indicates that small amounts of pyrrhotite $\left(\mathrm{Fe}_{7} \mathrm{~S}_{8}\right)^{1}$, troilite $(\mathrm{FeS})^{2}$, greigite $\left(\mathrm{Fe}_{3} \mathrm{~S}_{4}\right)^{3}, \mathrm{pyrite}\left(\mathrm{FeS}_{2}\right)^{4}$, magnetite $\left(\mathrm{Fe}_{3} \mathrm{O}_{4}\right)^{5}$, and hematite $\left(\mathrm{Fe}_{2} \mathrm{O}_{3}\right)^{6}$ phases are present. Table $\mathrm{S} 1$ contains the results obtained from the Rietveld analysis of the iron sulfides. Cell parameters of mackinawite (the predominant phase) for different FeS samples are shown in the Table S2. A variation in the cell parameters was observed for all the samples after the refinement. Literature indicates that this variation could possibly arise from the intercalation of the water molecules between the tetrahedral layers and the lattice relaxation due to small crystal size during the synthesis process. ${ }^{7}$

Table S1. Rietveld analysis of powder X-ray diffraction pattern of iron sulfide samples synthesized using different surfactant and iron sources.

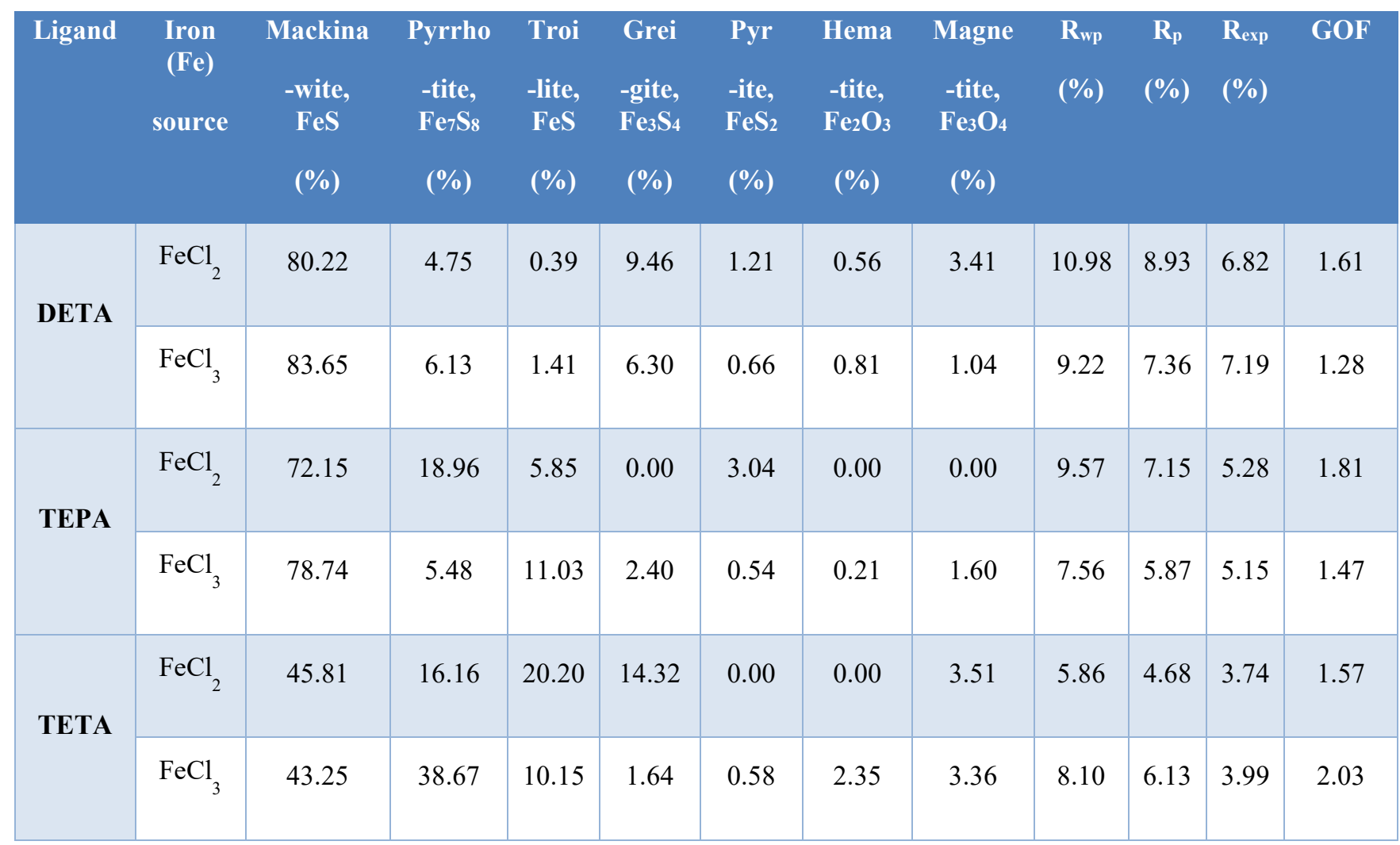


Table S2. Cell parameters of mackinawite phase (the predominant phase in the sample) obtained by Rietveld analysis of powder Xray diffraction pattern of iron sulfide samples synthesized using different ligand and iron sources.

\begin{tabular}{|c|c|c|c|c|c|c|c|}
\hline Cell & Before & \multicolumn{6}{|c|}{ After refinement } \\
\hline parameters & refinement & D2 & D3 & $\mathrm{P} 2$ & P3 & $\mathrm{T} 2$ & $\mathrm{~T} 3$ \\
\hline$a, b(\AA)$ & 3.6735 & $\begin{array}{c}3.5157 \\
(63)\end{array}$ & $\begin{array}{c}3.8830 \\
(100)\end{array}$ & $\begin{array}{c}3.8421 \\
(29)\end{array}$ & $\begin{array}{c}3.7309 \\
(65)\end{array}$ & $\begin{array}{c}3.7340 \\
(850)\end{array}$ & $\begin{array}{c}4.0807 \\
(62)\end{array}$ \\
\hline$c(\AA)$ & 5.0328 & $\begin{array}{c}5.1168 \\
(8)\end{array}$ & $\begin{array}{c}5.3850 \\
(110)\end{array}$ & $\begin{array}{c}4.8392 \\
(36)\end{array}$ & $\begin{array}{c}5.0428 \\
(74)\end{array}$ & $\begin{array}{c}5.5940 \\
(940)\end{array}$ & $\begin{array}{c}5.1968 \\
(50)\end{array}$ \\
\hline$\alpha(=\beta=\gamma)\left(^{\circ}\right)$ & 90.0 & 90.0 & 90.0 & 90.0 & 90.0 & 90.0 & 90.0 \\
\hline
\end{tabular}


(a)
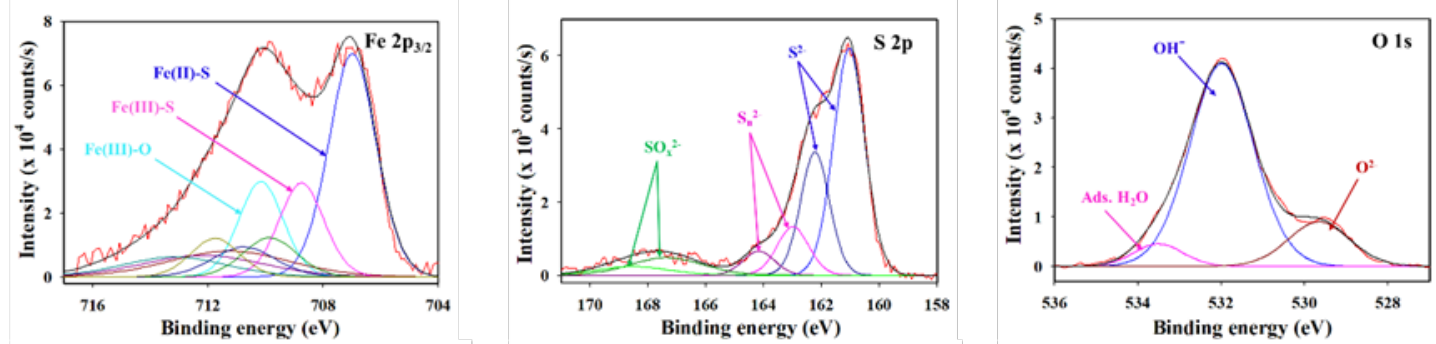

(b)
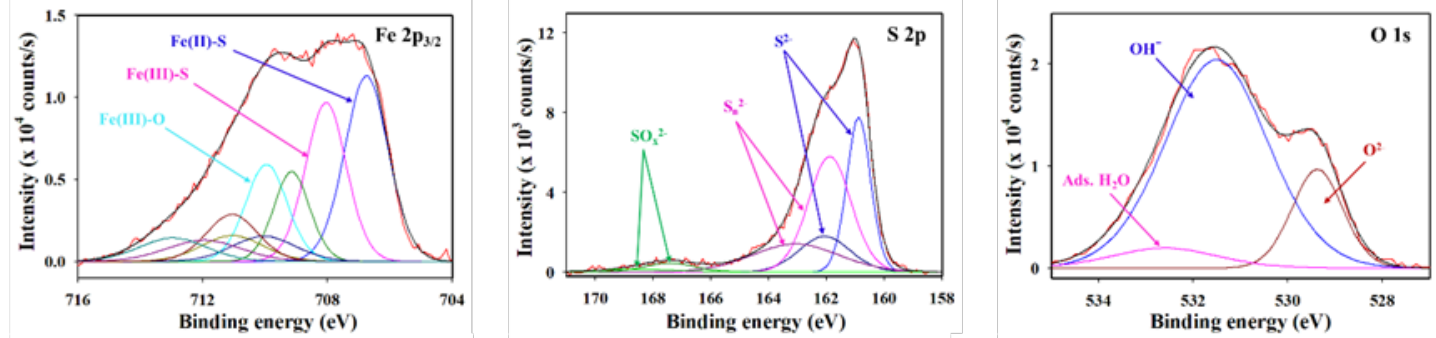

(c)
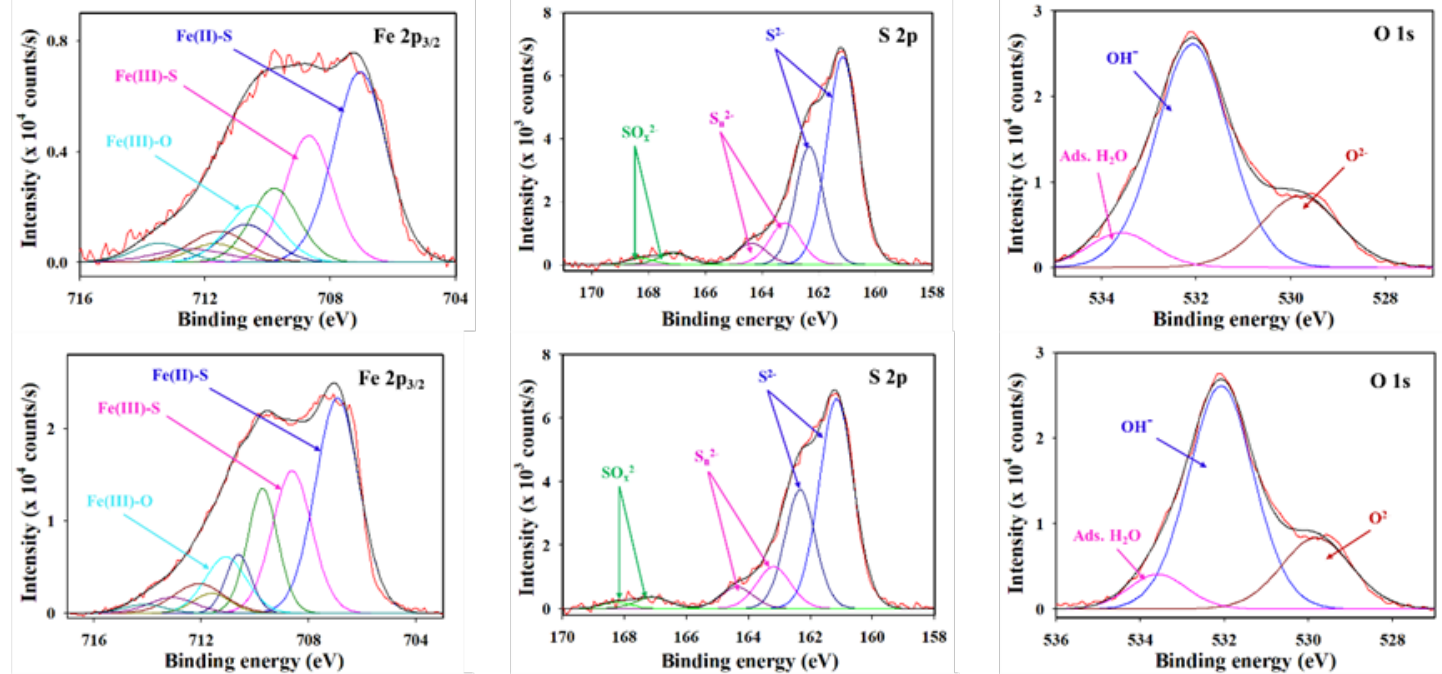

(d)
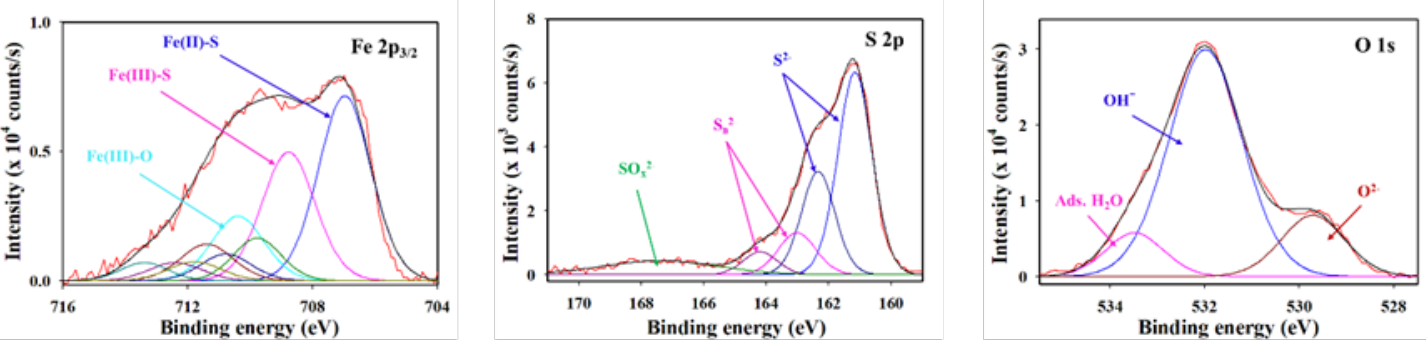

Figure S3. XPS spectra: Fe (2p3/2) region, S 2p region, and O 1s region of (a) D3, (b) P2, (c) P3, (d) T2, and (e) T3. 
Table S3. Summary of the measured and literature reported binding energies (BE) and peak areas for $\mathrm{Fe}(2 \mathrm{p} 3 / 2), \mathrm{S}(2 \mathrm{p})$ and $\mathrm{O}(1 \mathrm{~s})$ photoelectron spectra of FeS sample synthesized from $\mathrm{FeCl}_{2}$ and DETA (D2).

\begin{tabular}{|c|c|c|c|c|}
\hline \multirow[t]{2}{*}{ Chemical Species } & \multicolumn{2}{|c|}{ In this study } & \multicolumn{2}{|c|}{ Literature } \\
\hline & $\mathrm{BE}(\mathrm{eV})$ & Area (\%) & BE (eV) & References \\
\hline $\mathrm{Fe} 2 \mathrm{p}_{3 / 2}$ & 707.1 & 24.87 & 707.0 & Mycroft et al. $(1990)^{8}$ \\
\hline $\mathrm{Fe}(\mathrm{II})-\mathrm{S}$ & & & 707.1 & Pratt et al. $(1994 a, b)^{9,10}$ \\
\hline \multirow[t]{2}{*}{$\mathrm{Fe}(\mathrm{III})-\mathrm{S}$ major } & 709.4 & 22.39 & 709.2 & Pratt et al. $(1994 a, b)^{9,10}$ \\
\hline & & & 709.15 & Herbert et al. $(1998)^{11}$ \\
\hline \multirow[t]{3}{*}{ Fe(III)-S multiplets } & 710.3 & 14.93 & 710.3 & Pratt et al. $(1994 a, b)^{9,10}$ \\
\hline & 711.4 & 7.96 & 711.3 & \\
\hline & 712.4 & 5.97 & 712.2 & \\
\hline $\mathrm{Fe}(\mathrm{III})-\mathrm{O}$ major & 710.5 & 9.95 & 710.5 & Pratt et al. $(1994 a)^{9}$ \\
\hline \multirow[t]{3}{*}{$\mathrm{Fe}(\mathrm{III})-\mathrm{O}$ multiplets } & 711.4 & 5.97 & 711.6 & Pratt et al. $(1994 a)^{9}$ \\
\hline & 712.4 & 3.98 & 712.7 & \\
\hline & 713.3 & 3.98 & 713.8 & \\
\hline \multirow[t]{2}{*}{$\mathrm{S}^{2-}\left(2 \mathrm{p}_{3 / 2}\right)$} & 160.8 & 42.42 & 161.3 & Pratt et al. $(1994 a, b)^{9,10}$ \\
\hline & & & 160.9 & Herbert et al. $(1998)^{11}$ \\
\hline $\mathrm{S}^{2-}\left(2 \mathrm{p}_{1 / 2}\right)$ & 162.0 & 21.21 & 162.8 & Mullet et al. $(2002)^{12}$ \\
\hline $\mathrm{S}_{\mathrm{n}}^{2-}\left(2 \mathrm{p}_{3 / 2}\right)$ & 162.7 & 12.91 & 163.6 & Pratt et al. $(1994 a)^{9}$ \\
\hline $\mathrm{S}_{\mathrm{n}}^{2-}\left(2 \mathrm{p}_{1 / 2}\right)$ & 163.9 & 6.45 & 164.4 & Mullet et al. $(2002)^{12}$ \\
\hline $\mathrm{SO}_{\mathrm{x}}^{2-}$ & 167.6 & 17.01 & 168.1 & Thomas et al. $(1998)^{13}$ \\
\hline $\mathrm{O}^{2-}$ & 529.3 & 21.86 & 529.8 & Jones et al. $(1992)^{14}$ \\
\hline $\mathrm{OH}^{-}$ & 531.2 & 72.95 & 531.4 & McIntyre and Zetaruk (1977) \\
\hline Adsorbed $\mathrm{H}_{2} \mathrm{O}$ & 533.3 & 5.19 & 532.6 & Pratt et al. $(1996)^{16}$ \\
\hline
\end{tabular}




\section{REFERENCES}

(1) Lennie, A. R.; Redfern, S. A. T.; Schofield, P. F.; Vaughan, D. J. Synthesis and Rietveld Crystal Structure Refinement of Mackinawite,

Tetragonal FeS. Mineral. Mag. 1995, 59, 677-683. https://doi.org/10.1180/minmag.1995.059.397.10.

(2) Elliot, A. D. Structure of Pyrrhotite 5C ( $\left.\mathrm{Fe}_{9} \mathrm{~S}_{10}\right)$. Acta Cryst. B 2010, 66, 271-279. https://doi.org/10.1107/S0108768110011845.

(3) Skála, R.; Císařová, I.; Drábek, M. Inversion Twinning in Troilite. Am. Mineral. 2006, 91, 917-921. https://doi.org/10.2138/am.2006.1999.

(4) Rieder, M.; Crelling, J. C.; Šustai, O.; Drábek, M.; Weiss, Z.; Klementová, M. Arsenic in Iron Disulfides in a Brown Coal from the North Bohemian Basin, Czech Republic. Int. J. Coal Geol. 2007, 71, 115-121. https://doi.org/10.1016/j.coal.2006.07.003.

(5) Claassen, A. A. The Scattering Power of Oxygen and Iron for X-Rays. Proc. Phys. Soc. London 1925, 38, $482-487$. https://doi.org/10.1088/1478-7814/38/1/348.

(6) Rozenberg, G. Kh.; Dubrovinsky, L. S.; Pasternak, M. P.; Naaman, O.; Le Bihan, T.; Ahuja, R. High-Pressure Structural Studies of Hematite $\mathrm{Fe}_{2} \mathrm{O}_{3}$. Phys. Rev. B 2002, 65, 064112. https://doi.org/10.1103/PhysRevB.65.064112.

(7) Wolthers, M.; Van der Gaast, S. J.; Rickard, D. The Structure of Disordered Mackinawite. Am. Mineral. 2003, 88, $2007-2015$. https://doi.org/10.2138/am-2003-11-1245.

(8) Mycroft, J. R.; Bancroft, G. M.; McIntyre, N. S.; Lorimer, J. W.; Hill, I. R. Detection of Sulphur and Polysulphides on Electrochemically Oxidized Pyrite Surfaces by X-Ray Photoelectron Spectroscopy and Raman Spectroscopy. J. Electroanal. Chem. Interf. Electrochem. 1990, 292, 139-152. https://doi.org/10.1016/0022-0728(90)87332-E.

(9) Pratt, A. R.; Muir, I. J.; Nesbitt, H. W. X-Ray Photoelectron and Auger Electron Spectroscopic Studies of Pyrrhotite and Mechanism of Air Oxidation. Geochim. Cosmochim. Acta 1994, 58, 827-841. https://doi.org/10.1016/0016-7037(94)90508-8.

(10) Pratt, A. R.; Nesbitt, H. W.; muir, I. J. Generation of Acids from Mine Waste: Oxidative Leaching of Pyrrhotite in $\mathrm{Dilute}_{2} \mathrm{SO}_{4} \mathrm{Solutions}$ at PH 3.0. Geochim. Cosmochim. Acta 1994, 58, 5147-5159. https://doi.org/10.1016/0016-7037(94)90300-X.

(11) Herbert, R. B.; Benner, S. G.; Pratt, A. R.; Blowes, D. W. Surface Chemistry and Morphology of Poorly Crystalline Iron Sulfides Precipitated in Media Containing Sulfate-Reducing Bacteria. Chem. Geol. 1998, 144, 87-97. https://doi.org/10.1016/S0009-2541(97)00122-8.

(12) Mullet, M.; Boursiquot, S.; Abdelmoula, M.; Génin, J.-M.; Ehrhardt, J.-J. Surface Chemistry and Structural Properties of Mackinawite Prepared by Reaction of Sulfide Ions with Metallic Iron. Geochim. Cosmochim Acta 2002, 66, 829-836. https://doi.org/10.1016/S00167037(01)00805-5.

(13) Thomas, J. E.; Jones, C. F.; Skinner, W. M.; Smart, R. St. C. The Role of Surface Sulfur Species in the Inhibition of Pyrrhotite Dissolution in Acid Conditions. Geochim. Cosmochim. Acta 1998, 62, 1555-1565. https://doi.org/10.1016/S0016-7037(98)00087-8.

(14) Jones, C. F.; LeCount, S.; Smart, R. St. C.; White, T. J. Compositional and Structural Alteration of Pyrrhotite Surfaces in Solution: XPS and XRD Studies. Appl. Surf. Sci. 1992, 55, 65-85. https://doi.org/10.1016/0169-4332(92)90382-8.

(15) McIntyre, N. S.; Zetaruk, D. G. X-Ray Photoelectron Spectroscopic Studies of Iron Oxides. Anal. Chem. 1977, 49, 1521-1529. https://doi.org/10.1021/ac50019a016.

(16) Pratt, A. R.; Nesbitt, H. W.; Mycroft, J. R. The Increased Reactivity of Pyrrhotite and Magnetite Phases in Sulphide Mine Tailings. J. Geochem. Explor. 1996, 56, 1-11. https://doi.org/10.1016/0375-6742(96)00008-8. 